\title{
Motion Tracking of Intelligent Surveillance based on Machine Vision and Infrared Spectroscopy
}

\author{
Xiaohua Zhang ${ }^{1}$, Bo Huang ${ }^{1}$ \\ ${ }^{1}$ Department of Computer Science and Technology, Chengdu Neusoft University, \\ SiChuan Chengdu, 611844, China
}

Keywords: Machine Vision; Infrared Spectroscopy; Intelligence Surveillance; Robot; Positioning Error; Kinematics Model

\begin{abstract}
Circumferential point method has been adopted in this paper for calibration. Binocular vision dynamic tracking technique is used to track the independent motion axis of each joint of the robot, and then construct and fit the axis of joint to attain kinematic model parameters. This method does not need previous complicated steps of numerous robot kinematics models iterating solution equation; for the independent-developed robot control system, the linkage model parameters attained from above method can offer feedback to the robot timely, execute parameter amendment and compensation, and then improve the positioning accuracy of robot. This method is with advantages of generality, momentarily and easy operation etc. Experiment has shown that through parameter feedback compensation, the positioning error of the independent-developed robot has been decreased significantly. This method is easy to realize, which provides reliable evidence for robot accuracy research.
\end{abstract}

\section{Introduction}

With the development of industrial automation, the robot has been applied in production more and more extensively. Generally speaking, the repeatability accuracy of robot is high, but its absolute accuracy is relatively low, which will cause great influence on the using performance of the robot. For the robot without calibration, the accuracy error can be few millimeters. Therefore, it has to make accurate calibration for robot in a lot of applications. Calibration is the integration process of modeling, measurement, parameter identification and error compensation ${ }^{[1]}$. The purpose of calibration is to decrease the geometric parameter error of robot. Geometric parameter error of robot is the error deviation between nominal value and true value of geometric parameter in kinematics model. The kinematics calibration of geometric parameters of robot connecting rod involves in the conversion problem between measurement coordinate system and robot base coordinate. Some calibration processes establish the coordinate system of measuring instrument on the base coordinate system of robot, but this will cause complicated coordinate system conversion, make the calculation process complicated and even difficult to solve ${ }^{[2 \sim 4]}$.

\section{Binocular Vision Dynamic Tracking Technique}

The main function of binocular vision dynamic tracking technique is to detect moving target automatically and realize real-time tracking and three-dimensional ranging. The basic skills are as shown in figure 1.

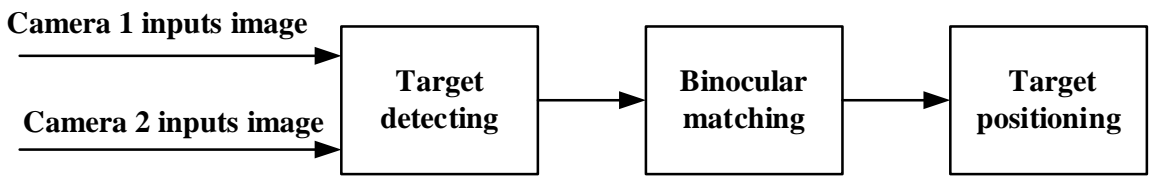

Fig.1. Binocular vision dynamic tracking steps

Basic principle of binocular vision positioning system is as shown in figure 2 . With the adoption of parallax formed by the same feature point $P$ at left and right images, it can measure the depth information of feature point. 


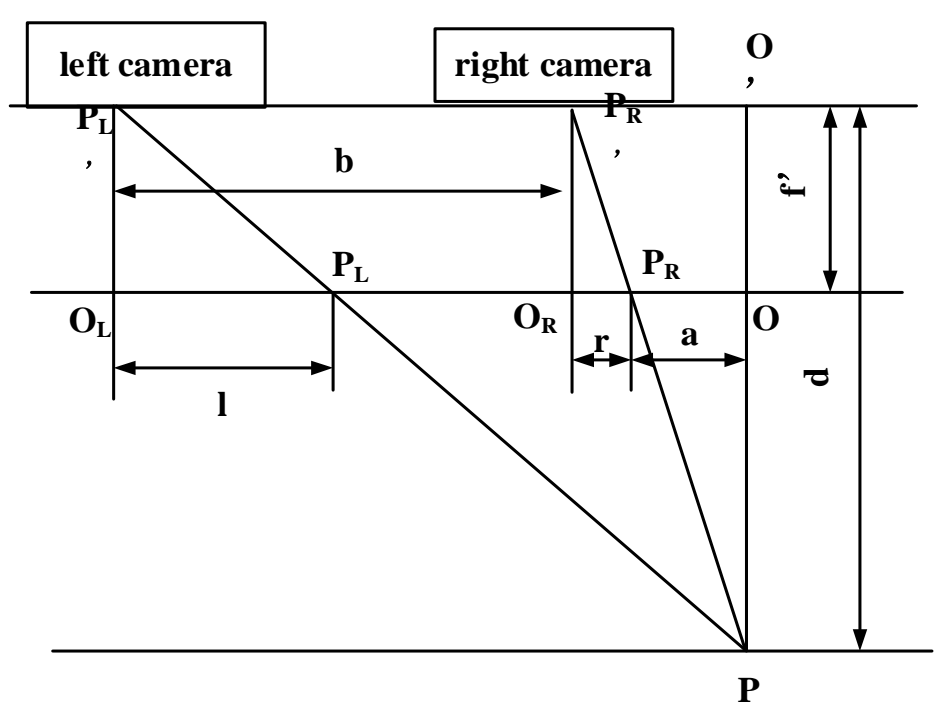

Fig.2. Schematic diagram of binocular vision positioning system

With the parameter value (internal parameter and external parameter) of video camera, the two-dimensional image coordinate of the surface point of the measured object can be converted into three-dimensional world coordinate system. The calculation formula is as shown in the following:

$$
Z_{c}\left[\begin{array}{c}
U \\
V \\
0
\end{array}\right]=\left[\begin{array}{cccc}
\frac{f}{d_{x}} & 0 & U_{0} & 0 \\
0 & \frac{f}{d_{y}} & V_{0} & 0 \\
0 & 0 & 1 & 0
\end{array}\right]\left[\begin{array}{cc}
R & T \\
O^{T} & 1
\end{array}\right]\left[\begin{array}{c}
X_{w} \\
Y_{w} \\
Z_{w} \\
1
\end{array}\right]
$$

In formula (1), $Z_{c}$ is the vertical distance from object surface feature point to optical center plane of left and right camera; $\left(x_{w}, y_{w}, z_{w}\right)$ is the coordinate of world coordinate system of feature point; $\left(U_{0}, V_{0}\right)$ is the pixel coordinate of image center; $(U, V)$ is the coordinate of image coordinate system of measured point. $d_{x}$ and $d_{y}$ are the physical distance between adjacent pixels on horizontal and vertical directions. $R$ is $3 \times 3$ rotation matrix and T is a translation vector ${ }^{[12]}$.

Binocular dynamic tracking system is with two high-quality optics CCS and special light source. Within the operation range, measure reflecting target automatically. The system calculates the 3D coordinate of target in real time automatically based on the trigonometric measurement principle and transfers the confirmed coordinate value to the software for following processing. The measurement accuracy of this system within the measurement range is as high as $0.022 \mathrm{~mm}$, single point repeatability accuracy is $0.045 \mathrm{~mm}$, volume accuracy is $0.075 \mathrm{~mm}$ and dynamic tracking speed is $30 \mathrm{~Hz}$.

\section{Kinematics Characteristics of Robot}

\section{Kinematics characteristics of robot}

The research object of this paper is independent-developed 6R industrial robot and the basic structure is as shown in figure 3. The free degree of the robot is 6 and it is with rotation joints completely. The first three joints Axis 1, Axis 2 and Axis 3 control the position of the end wrist of robot, while the last three joints Axis 4, Axis 5 and Axis 6 control the posture of the end wrist of robot. The specific joint structure of robot consists of rotation main body (lumbar joint), large arm (shoulder joint), small arm (elbow joint) and wrist part (wrist joint) etc. 


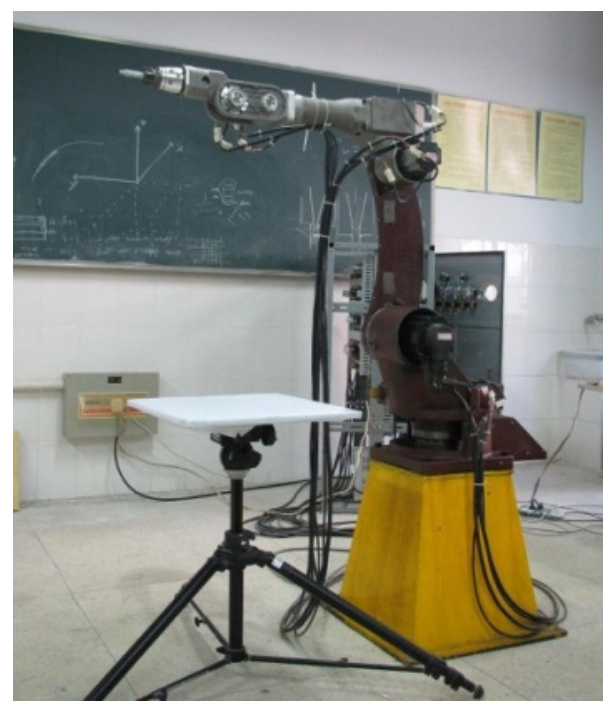

Fig.3. Independent-developed robot structure

This robot linkage system can be regarded as an open kinematic chain, which is formed by a series of connecting rods through rotating joints in series. One end of the open chain is fixed at the based and the other end is free. Installation tool (or end performer) operates object and accomplish various operations. The joint is driven by the driver. The relative movement of joint cause the movement of connecting rod, which makes the gripper, reaches the requested posture. To study the posture relationship between connecting rods, it can fix a coordinate system at each connecting rod and then describe the relationship between these coordinate systems. This paper adopts coordinate system method proposed by Denavit and Hartenberg to attain the kinematics model of the robot, as shown in figure 4. Robot theory connecting rod parameters are as shown in table 1.

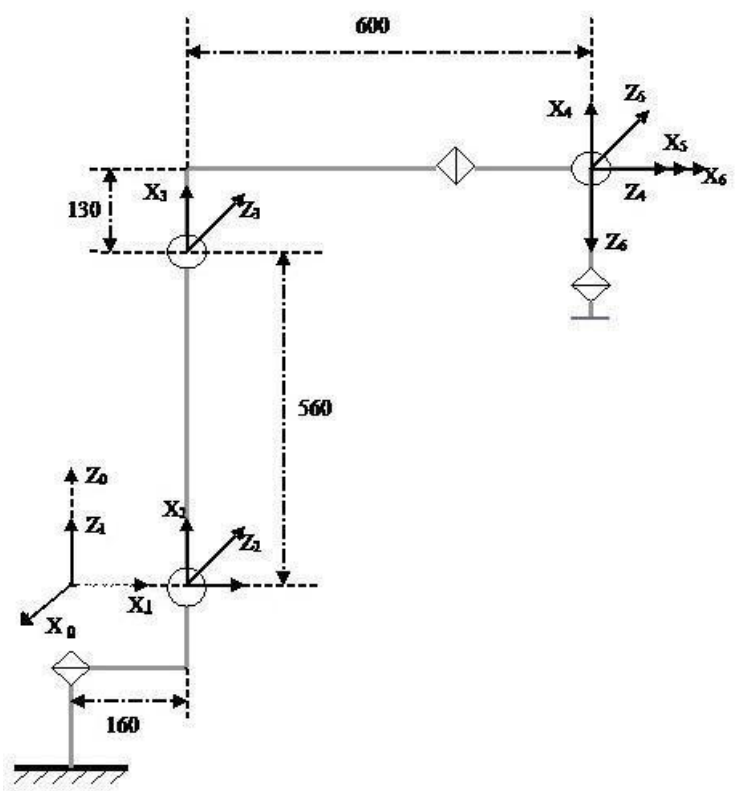

Fig.4. Robot D-H model

Table 1 Robot theory kinematics parameters

\begin{tabular}{|c|c|c|c|c|c|}
\hline connecting rod $\mathrm{i}$ & $\theta_{\mathrm{i}}\left({ }^{\circ}\right)$ & $\begin{array}{c}\alpha_{\mathrm{i}-1} \\
\left({ }^{\circ}\right)\end{array}$ & $\begin{array}{c}\mathrm{a}_{\mathrm{i}-1} \\
(\mathrm{~mm})\end{array}$ & $\begin{array}{c}\mathrm{d} \\
(\mathrm{mm})\end{array}$ & Range of joint angle $\left(^{\circ}\right)$ \\
\hline 1 & $\theta_{1}\left(90^{\circ}\right)$ & $0^{\circ}$ & 0 & 0 & $-170 \sim+170$ \\
\hline 2 & $\theta_{2}\left(-90^{\circ}\right)$ & $-90^{\circ}$ & 160 & 0 & $-170 \sim+20$ \\
\hline 3 & $\theta_{3}\left(0^{\circ}\right)$ & $0^{\circ}$ & 560 & 0 & $-60 \sim+70$ \\
\hline 4 & $\theta_{4}\left(0^{\circ}\right)$ & $-90^{\circ}$ & 130 & 600 & $-170 \sim+170$ \\
\hline 5 & $\theta_{5}\left(90^{\circ}\right)$ & $90^{\circ}$ & 0 & 0 & $-90 \sim+140$ \\
\hline 6 & $\theta_{6}\left(0^{\circ}\right)$ & $-90^{\circ}$ & 0 & 0 & $-180 \sim+180$ \\
\hline
\end{tabular}


Transform formula of robot connecting rod is:

$$
{ }^{i-1} T_{i}=\left[\begin{array}{cccc}
c \theta_{i} & -s \theta_{i} & 0 & a_{i-1} \\
s \theta_{i} c \alpha_{i-1} & c \theta_{i} c \alpha_{i-1} & -s \alpha_{i-1} & -d_{i} s \alpha_{i-1} \\
s \theta_{i} s \alpha_{i-1} & c \theta_{i} s \alpha_{i-1} & c \alpha_{i-1} & d_{i} c \alpha_{i-1} \\
0 & 0 & 0 & 1
\end{array}\right]
$$

In the formula, s is sine of angle $\theta$ and c is the cosine of angle $\theta$.

Multiply change of connecting rod to attain change matrix of manipulator:

${ }_{6}^{0} \mathbf{T}={ }_{1}^{0} T_{2}^{1} T_{3}^{2} T_{4}^{3} T_{5}^{4} T_{6}^{5} T$

That is robot end performer posture matrix.

\section{Robot positioning error}

The deviation of robot geometric parameter between nominal value and true value, use $\Delta a i, \Delta d i$, $\Delta \alpha i, \Delta \theta i$ to express the length deviation of connecting rod, connecting rod offset, twist angle deviation and joint angle deviation, in which $\Delta a i$ and $\Delta d i$ are the bar length errors because of processing accuracy and during robot assembling. $\Delta \alpha i$ is angle error aroused by parallelism and perpendicularity between adjacent axes. $\Delta \theta i$ is zero offset effort caused by misalignment between zero position of angle optical encoder and zero position of joint rotation in nominal model in the robot assembly process. These geometric parameter errors have great impact on the positioning accuracy of robot end performer.

The robot end posture can be attained based on the robot geometric parameter. Use $\boldsymbol{P}^{n}$ to express the theoretical position of robot end flange center point in robot base coordinate system; use $\boldsymbol{P}^{r}$ to express actual end posture and use $\Delta \boldsymbol{P}$ to express the positioning error of robot end posture.

$$
\begin{aligned}
& P^{n}=F(a, d, \alpha, \theta) \\
& P^{r}=F(a+\Delta a, d+\Delta d, \alpha+\Delta \alpha, \theta+\Delta \theta)
\end{aligned}
$$

Positioning error can be attained from formula (2) and (3):

$\Delta \boldsymbol{P}=\boldsymbol{P}^{r}-\boldsymbol{P}^{n}$

According to the connecting rob parameter model in the independent-developed robot control system, current stage only considers about the robot end TCP location error.

\section{Robot calibration experiment}

Method proposed in this paper has been adopted to make calibration experiment for independent-developed 6R joint robot. Parameter feedback compensation has been made for the robot through measuring the actual $\mathrm{D}-\mathrm{H}$ parameters of robot. In the following, it measures the robot positioning error under designed $\mathrm{D}-\mathrm{H}$ parameters as well as the robot positioning error under actual $\mathrm{D}-\mathrm{H}$ parameters, and then makes comparative analysis of these two sets of positioning errors. Calibration experiment mainly consists of four steps, including robot joint coordinate system acquisition, robot parameter identification, robot parameter feedback compensation and experimental result analysis.

\section{Robot joint coordinate system acquisition}

Robot joint coordinate system acquisition involves in robot base coordinate system. Due to the adoption of high-accuracy dynamic tracking system, it can be found in the measurement process that the plane where the joint motor axis circle formed by independent rotation of the $1^{\text {st }}$ joint is can replace the plane where is the first joint of robot ontology structure with high accuracy. Based on this, it can construct robot base coordinate system. The construction principle is that under robot initial zero state, it takes the normal of the $1^{\text {st }}$ joint plane as the $\mathrm{Z}$ axis direction of base coordinate system, takes the normal of the second joint plane as the $\mathrm{X}$ axis direction of base coordinate system, while the $\mathrm{Y}$ direction will be generated automatically, which meets coordinate system right hand rule: $Y=Z \times X$. With the adoption of robot single joint independent movement, it can attain each joint circle and then attain the actual relationship of each joint circle space of the robot. Finally, based on D-H modeling method, it can construct robot joint coordinate system. The robot parameter model is as shown in figure 5. 


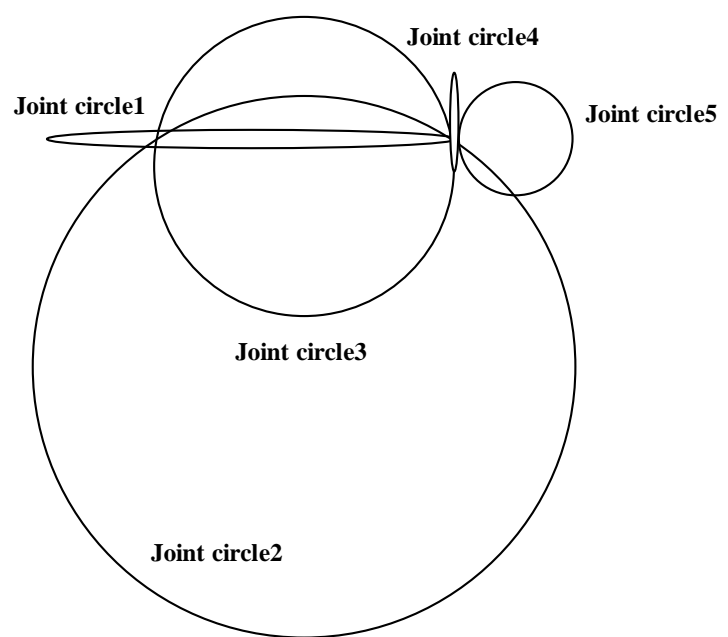

\section{Robot parameter feedback compensation}

Fig.5. Constructed robot parameter model

The actual connecting rod parameter of robot can be attained from above experimental results. This paper combines robot control system and offers the average value of identified parameter to robot, and then modifies the robot kinematics model parameters of control system and accomplishes parameter feedback compensation of robot calibration process. Before and after parameter compensation, it makes dynamic tracking for robot. Considering about the deviation between actual TCP of robot and the TCP position in robot theory kinematics model, this paper constructs robot base coordinate system and coordinate system of actual robot TCP respectively. Robot actual base coordinate system and actual TCP coordinate relationship is established dynamically through actual measurement results of binocular vision system and then track robot accurately.

(1) Before parameter feedback compensation, control the robot to reach any position in its working space. Adopt binocular vision dynamic tracking system to track robot TCP, measure the coordinate of any position that TCP reaches in the working space, compare the attained position coordinate with theory position coordinate of robot control system and then it can get the position error before compensation.

(2) And then adopt the same tracking method, after making parameter feedback compensation for robot kinematics, track and measure the coordinate of robot TCP reaching any position of the working space, and then attain the position error after compensation. The positioning error before and after parameter compensation is as shown in table 5

\section{Experimental result analysis}

This paper adopts the principle of dynamic tracking measurement as well as binocular vision dynamic tracking system to make calibration for independent-developed robot. It can be seen from calibration experimental results that

(1) In robot kinematics model, there is big error between theoretical value and actual measured value of connecting rod parameter, which is mainly presented in data. The nominal value of design is $130 \mathrm{~mm}$ and then the data average value measured by actual dynamic measurement method is $126.449 \mathrm{~mm}$. The modification of this parameter has improved the positioning accuracy of robot greatly.

(2) It can be seen from the TCP coordinate data of robot actual end performer that the $\mathrm{x}$ coordinate value of TCP in theory is 0 , but due to the existence of numerous influencing factors, such as processing error and assembly error etc, it is easy to cause position error of robot actual end TCP for robot base coordinate system. During the tracking, depending on the dynamic tracking of binocular vision system as well as the advantages of dynamic modeling, this paper sets up the relationship between robot base coordinate similar to reality and actual TCP coordinate.

(3) It can be seen from positioning error data that the positioning accuracy of robot after compensation has been improved significantly. The average error of positioning accuracy of robot before compensation is $3.997 \mathrm{~mm}$ and the standard variance is 0.189 ; the average error of positioning accuracy after compensation is $1.067 \mathrm{~mm}$ and the standard variance is 0.104 . The 
average positioning error has increased by 3.7 times and the average error has improved by 1.8 times.

\section{Conclusion}

In the process of developing industrial robot independently, for the accuracy problem referring to robot, this paper adopts dynamic tracking and dynamic modeling method and depends on binocular vision dynamic tracking technique to make kinematic calibration for robot. It makes correction compensation on the basis of analyzing robot theory kinematics parameter and then improves the positioning accuracy of robot. The experiment program designed in this paper is easy to measure several robot kinematic parameters. The method of constructing joint axis by dynamic detection of independent joint movement can avoid the measurement error caused by mechanical structural error or contact measurement. It is with high reliability; moreover, the experiment program designed in this paper is with good practicability in robot accuracy measurement aspect.

\section{Acknowledgement}

Research project of Sichuan Provincial Education Department (17ZB0007).

\section{References}

[1] Yingyue Zhang, Jennifer W. Chan, Alysha Moretti, and Kathryn E. Uhrich, Designing Polymers with Sugar-based Advantages for Bioactive Delivery Applications, Journal of Controlled Release, 2015, 219, 355-368.

[2] Yingyue Zhang, Qi Li, William J. Welsh, Prabhas V. Moghe, and Kathryn E. Uhrich, Micellar and Structural Stability of Nanoscale Amphiphilic Polymers: Implications for Anti-atherosclerotic Bioactivity, Biomaterials, 2016, 84, 230-240.

[3] Jennifer W. Chan, Yingyue Zhang, and Kathryn E. Uhrich, Amphiphilic Macromolecule Self-Assembled Monolayers Suppress Smooth Muscle Cell Proliferation, Bioconjugate Chemistry, 2015, 26(7), 1359-1369.

[4] Dalia S. Abdelhamid, Yingyue Zhang, Daniel R. Lewis, Prabhas V. Moghe, William J. Welsh, and Kathryn E. Uhrich, Tartaric Acid-based Amphiphilic Macromolecules with Ether Linkages Exhibit Enhanced Repression of Oxidized Low Density Lipoprotein Uptake, Biomaterials, 2015, 53, 32-39.

[5] Yingyue Zhang, Jennifer W. Chan, Alysha Moretti, and Kathryn E. Uhrich, Designing Polymers with Sugar-based Advantages for Bioactive Delivery Applications, Journal of Controlled Release, 2015, 219, 355-368. 